\title{
A prospective randomised radiostereometric analysis trial of SmartSet HV and Palacos $R$ bone cements in primary total hip arthroplasty
}

\author{
Otto S. Husby • Kristin Haugan · Pål Benum • \\ Olav A. Foss
}

Received: 29 September 2009/ Accepted: 30 January 2010/Published online: 3 March 2010

(c) Springer-Verlag 2010

\begin{abstract}
Background Introduction of new bone cements into clinical practice should include radiostereometric studies.

Materials and methods A prospective randomised radiostereometric study was performed, comparing SmartSet HV and Palacos R acrylic bone cements (without antibiotics) using third-generation cementing techniques in primary total hip arthroplasty. Thirty-five patients (36 hips) undergoing Charnley total hip arthroplasty were randomised to receive either of the two cements and were followed with repeated clinical, radiographic and radiostereometric examinations over 24 months. Twenty-seven patients (28 hips) attended 2 years postoperatively.

Results The mean distal translation observed was $-0.15 \mathrm{~mm}$ for SmartSet HV and $-0.16 \mathrm{~mm}$ for Palacos R. The mean rotation around the longitudinal axis was $0.9^{\circ}$ for SmartSet HV and $1.2^{\circ}$ for Palacos R. The Merle d'Aubigne Postel score was the maximum of 18 points for all patients in both groups.

Conclusions No statistically significant difference in stem fixation with use of SmartSet HV and Palacos R was found at 2-year follow-up.
\end{abstract}

Keywords SmartSet HV · Palacos R - RSA - Charnley · THR

O. S. Husby · P. Benum · O. A. Foss

Orthopaedic Department, St. Olavs Hospital,

Trondheim University Hospital, 7005 Trondheim, Norway

O. S. Husby · K. Haugan $(\bowtie) \cdot$ P. Benum · O. A. Foss

Norwegian Orthopaedic Implant Research Unit,

St. Olavs Hospital, Bevegelsessenteret,

7006 Trondheim, Norway

e-mail: kristin.haugan@ntnu.no

\section{Introduction}

Many factors influence the long-term performance of cemented total hip replacement (THR) such as patient characteristics, the prosthetic components, bone cements and surgical techniques [1].

Increased prosthetic migration has been correlated with early loosening [2,3]. This was clearly demonstrated when using Boneloc cement (Biomet, Warsaw, Indiana). This low-viscosity cement was found to be catastrophic based on a randomised clinical study using radiostereometric analysis (RSA) [4] and 5 years of data from the Norwegian Arthroplasty Register [5].

The RSA technique enables calculation of the threedimensional translational and rotational movements of the implant relative to the bone with high precision and accuracy [6] and has become the gold standard for clinical evaluation of new surgical techniques and implants [7]. RSA is part of the recommended stepwise introduction of new surgical techniques and implants [8].

Fixation of the Charnley stem with Palacos R cement was previously found to be a good combination [9]. Although this manufacturer (Schering Plough, Belgium) no longer produces Palacos R, this cement was well established within the cement market, with many years of clinical success, at the time of conducting this clinical study.

The SmartSet HV (DePuy CMW, Blackpool, UK) is a high-viscosity bone cement, which is self-curing and composed of methyl methacrylate (monomer) and methyl methacrylate/methylacrylate copolymer (polymer) (PMMA). This cement guarantees, according to the manufacturer, a "buffer zone" of viscosity prior to and after implant insertion, achieved by using a combination of two methyl methacrylate-methyl acrylate copolymers. 
Such a buffer zone may contribute to more controlled pressurisation during implant insertion.

Preclinical mechanical tests revealed no differences in mechanical properties between Palacos R and SmartSet GHV (SmartSet HV with gentamicin added) when tested at $20^{\circ} \mathrm{C}$, whereas no comparisons at $37^{\circ} \mathrm{C}$ were made [10].

The objective of this prospective randomised study was to investigate early migration of the Charnley femoral prosthesis when implanted with either SmartSet HV or Palacos R bone cement. Neither of the cements contained an antibiotic.

\section{Materials and methods}

The study was conducted in accordance with the Declaration of Helsinki and approved by the Norwegian Technical and Scientific University Central Region Ethics Committee (reference 094-02).

The patients included were recruited from patients consecutively admitted to a single study centre (Orthopaedic Department, St. Olavs Hospital, Trondheim University Hospital Norway). Excluded from participation were any patients with an existing condition such as malignancy, pregnancy, severe osteoporosis and disabling musculoskeletal problems (other than in the hips), patients on corticosteroid treatment and patients who had already participated in a clinical study with an investigational product in the last 6 months.

Thirty-five patients were asked to participate, and all agreed. All patients signed an informed consent. Thirty-six hips (35 patients) were included (Table 1).

The Merle d'Aubigne Postel score [11] was recorded preoperatively as a baseline clinical evaluation of the patient's level of pain, mobility and walking ability. Absence of pain, mobility of greater than $90^{\circ}$ flexion and $30^{\circ}$ abduction, and normal, unlimited walking ability gives a maximum score of 18 points.

The cement randomization was electronically generated preoperatively (SAS version 8), with equal numbers of patients randomly allocated to each of the two cements. No stratification was used. Each code was kept in a sealed opaque envelope and broken during the operation, immediately before cementing. The single patient with bilateral hips included was randomised to receive the Palacos $\mathrm{R}$ cement in both.

Table 1 Clinical characteristics of each group

\begin{tabular}{|c|c|c|}
\hline & SmartSet HV & Palacos R \\
\hline \multirow[t]{2}{*}{ Hips included $(n)$} & 18 & 18 \\
\hline & Male (4), female (14) & Male (8), female (10) \\
\hline \multirow[t]{3}{*}{ Diagnosis $(n)$} & 16 Primary osteoarthritis & 17 Primary osteoarthritis \\
\hline & 1 Congenital hip dysplasia & 1 Avascular necrosis \\
\hline & 1 Post-traumatic arthritis & \\
\hline Age at surgery, years $(n=36)$ & $69(57-78)$ & $69(59-77)$ \\
\hline \multicolumn{3}{|l|}{ Mean (range) } \\
\hline Weight (include patients), $\mathrm{kg}$ & $75(60-90)$ & $78(52-98)$ \\
\hline \multicolumn{3}{|l|}{ Mean (range) } \\
\hline \multirow[t]{3}{*}{ Lost to follow-up $(n)$} & 4 & 4 \\
\hline & Missing baseline RSA examination (2) & Missing baseline RSA examination (2) \\
\hline & Death of patient before 2-year follow-up (2) & Did not attend arranged RSA examinations (2) \\
\hline \multirow[t]{3}{*}{ Returning for follow-up $(n)$} & 3 months $(n=14)$ & 3 months $(n=13)$ \\
\hline & 6 months $(n=10)$ & 6 months $(n=10)$ \\
\hline & 1 year $(n=16)$ & 1 year $(n=16)$ \\
\hline \multirow[t]{2}{*}{ Hips attending at 2-year follow-up (n) } & 14 & 14 \\
\hline & Male (2), female (12) & Male (5), female (9) \\
\hline \multirow[t]{2}{*}{ Diagnosis at 2-year follow-up $(n)$} & 13 Primary osteoarthritis & 13 Primary osteoarthritis \\
\hline & 1 Congenital hip dysplasia & 1 Avascular necrosis \\
\hline Age at surgery, years $(n=28)$ & $69(62-77)$ & $70(59-74)$ \\
\hline \multicolumn{3}{|l|}{ (2-year follow-up population) } \\
\hline \multicolumn{3}{|l|}{ Mean (range) } \\
\hline Weight at surgery, $\mathrm{kg}(n=28)$ & $74(60-85)$ & $76(52-90)$ \\
\hline \multicolumn{3}{|l|}{ (2-year follow-up population) } \\
\hline Mean (range) & & \\
\hline
\end{tabular}


One experienced surgeon (O.S.H.) performed the surgical intervention on all 36 hips, from October 2002 to October 2003.

The surgeon was not blinded in respect to the cement used. This was impossible due to the differences in colour and handling of the two types of cements. The patients were blinded to which cement they received.

The RSA technique employed requires tantalum beads fixed on the implant and beads implanted into the bone.

All patients received a Charnley flanged 40 prosthesis (DePuy International, Leeds UK), manufactured with tantalum bead mounted stainless-steel towers; one tower was fixed to the distal tip of the stem and one in the proximal shoulder region. A third marker is referenced by the central point of the Charnley 22.225-mm-diameter femoral head.

A posterolateral surgical approach (the hospital standard) was used, with a lateral incision [12]. A neck resection guide was used, and the femoral canal was entered through the piriformis fossa. The femur was prepared with a $12-\mathrm{mm}$ central reamer, followed by a $1-\mathrm{mm}$ all-direction oversized broach, and rinsed with saline using pulsed lavage. Bleeding was controlled at the prepared surfaces with use of a $1 \%$ adrenalin-soaked sponge.

Nine tantalum beads $(0.8 \mathrm{~mm}$ in diameter $)$ were implanted into the bone inside the prepared femoral cavity, ideally five in the greater trochanter and four in the lesser trochanter region. Third-generation cementing technique incorporating retrograde filling and proximal occlusion during pressurisation was used for all patients. Distal cement restriction was obtained using a polyethylene plug (Cement Restrictor, DePuy International, Leeds, UK). Palacos $\mathrm{R}$ was prechilled for minimum $24 \mathrm{~h}$ to $8^{\circ} \mathrm{C}$ before mixing. SmartSet HV was stored at $21^{\circ} \mathrm{C}$. Both cements were mixed under vacuum, using the Cemvac system (DePuy CMW, Blackpool UK) and a syringe and gun.

All patients received the standard Charnley Ogee cup (DePuy International, Leeds, UK). The same cement type was used for both the femoral and acetabular component.

Standard radiographic examination (AP and lateral view) of the hips was carried out.

Implant position relative to the central axis of the femur was described by manually measuring the alignment of the central axis of the stem with respect to the axis of the femoral canal. Discrepancies of greater than $\pm 5^{\circ}$ from the neutral position would be classified as malalignment.

The baseline RSA radiographic examinations were carried out as soon as the patients were capable of weight bearing and walking after surgery, typically within 7 days.

RSA evaluation was performed by using UmRSA software (version 6.0, RSA Biomedical Innovation, Umea, Sweden). The mean error fitting upper limit was set to $0.25 \mathrm{~mm}$, and the upper limit for the condition number 150 .

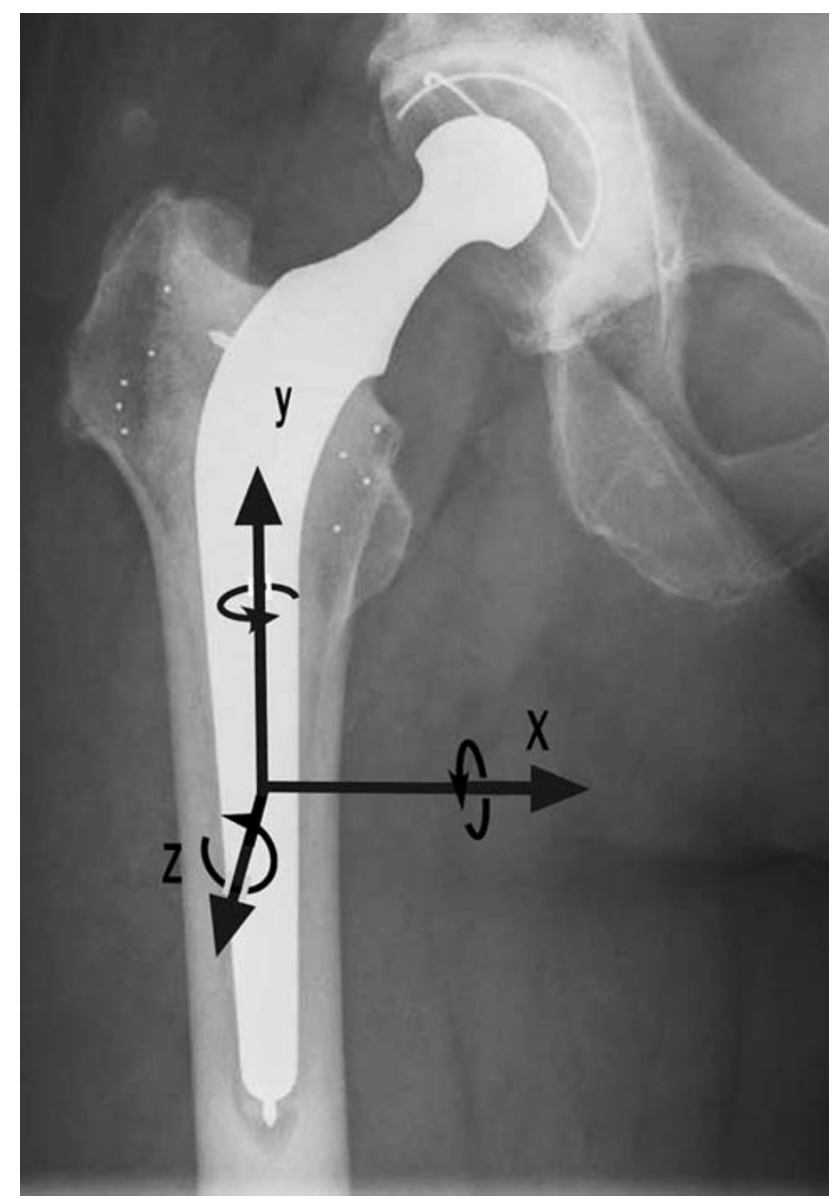

Fig. 1 The Charnley stem, labelled with the axes and directions of the rotations used. Tantalum markers embedded in the bone and mounted on the femoral stem are visible

The coordinate axes and directions of the rotations are illustrated in Fig. 1.

RSA operator was not blinded to the cement type used. We believe this to be acceptable. The RSA software automatically identifies markers and thereby reduces possible operator-induced biases.

The patients returned for postoperative follow-up at 3,6, 12 and 24 months. At each follow-up, standard radiographs, RSA and completion of the Merle d'Aubigne Postel score were recorded.

Only ten hips in each group attended at the 6-month RSA follow-up, and these RSA measurements were therefore excluded from publication due to the low number of participants.

\section{Statistical methods}

The sample size calculation was based on micromotion data of Charnley stems in combination with Palacos $\mathrm{R}$ cement earlier presented from our research laboratory [13] using 
Sample Power 2.0 (SPSS Inc., Chicago, USA). With sample size of 18 in each group, $\alpha=0.05$ and $\beta=0.80$, an alternative mean of more than $\pm 0.06 \mathrm{~mm}$ of migration and more than $\pm 0.92^{\circ}$ of rotation (translation along and rotations around the $Y$-axis) could be detected. An assumption of equal variance in both groups was made. Testing of normality of distribution was performed with Q-Q plots.

Two-tailed independent $t$-test was used to analyse differences in micromotion between the two cement groups. A probability level of $P<0.05$ indicated statistical significance.

The precision of the RSA technique was measured by double examinations of 22 patients at their 2-year followup visit. Each patient got up from the X-ray table and walked in the room between the two examinations, which were made within $10 \mathrm{~min}$ of each other.

The precision values were calculated as follows: first, the differences between the two examinations for each patient were calculated. Second, the standard deviation (SD) of these differences with respect to zero (not to the mean) was calculated [14].

$\mathrm{SD}=\sqrt{\frac{\sum_{i=1}^{n}\left(x_{i}\right)^{2}}{n}}$,

$X$ represents the differences between the double examinations $(n=22)$.

Finally, the SD multiplied by 2.074 (representing the 0.975 percentage point at a $t_{22}$ distribution) defines the precision.

\section{Results}

The micromotion measurement results are presented in Fig. 2 and further in Table 2.

The error fitting limits and conditions were met in all RSA analyses, and the measurement data were found to be normally distributed for all six data sets.

At 2-year follow-up there were no statistically significant differences in rotations or translations between the SmartSet HV group and the Palacos R group.

The precision data are presented in Table 3.

The Merle d'Aubigne Postel score was completed for each patient preoperatively and at 2-year follow-up. Preoperatively, the mean score and range was 10 (7-13) for the SmartSet HV group and 10 (7-12) for the Palacos R group. At 2 years, the score was the maximum of 18 points for all patients in both groups.

AP and lateral X-ray analysis indicated that all implants were positioned neutrally relative to the central axis of the femur. All cement mantles in both groups were intact and complete white-out in the bone interface was registered on all postoperative radiographs.
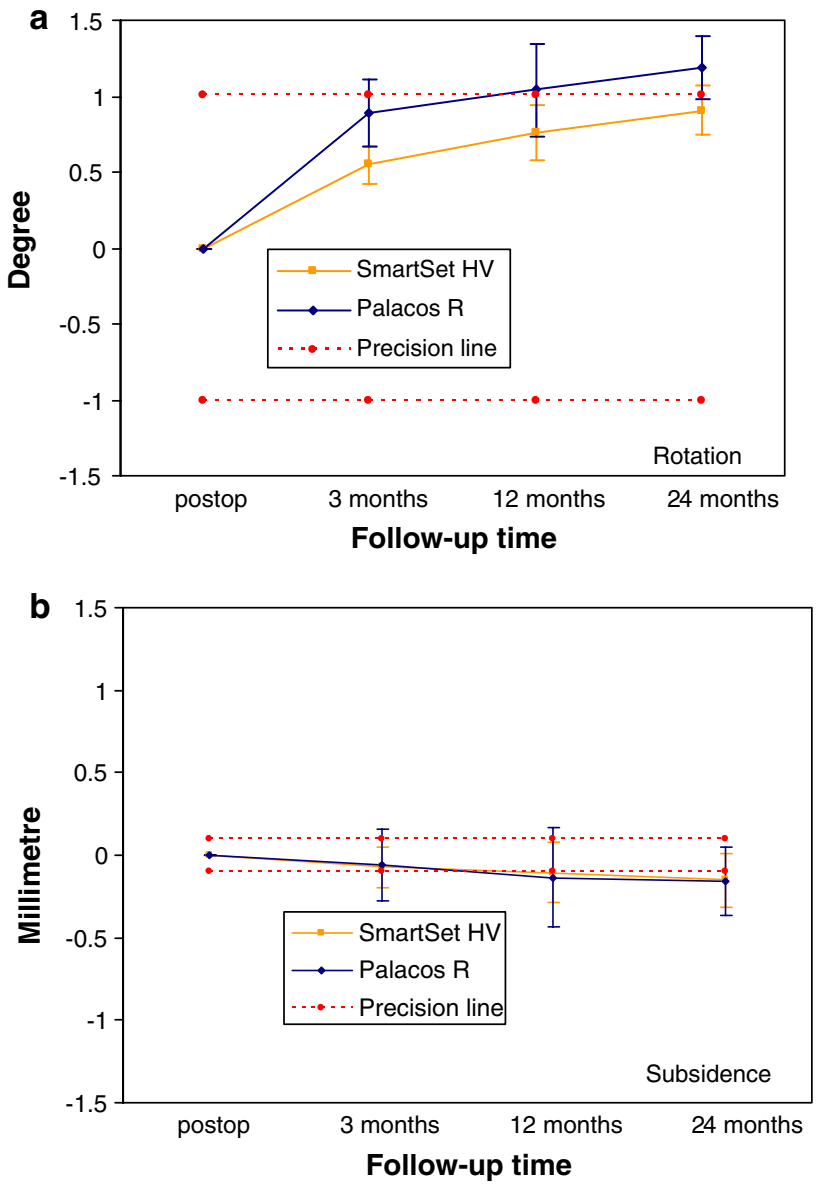

Fig. 2 (a) Rotation around the $Y$-axis. The graphs present mean and standard error of the mean. (b) Translation along the $Y$-axis. The graphs present mean and standard error of the mean

A total of three severe adverse events have been reported, none likely to be study related. One patient suffered from cerebral infarction, which occurred in the immediate postoperative period. This patient recovered fully and continues to be followed up in this investigation. Two patients died: one due to multi-organ failure, the other as a result of pneumonia. None of these patients had undergone a hip revision.

\section{Discussion}

Introduction of new orthopaedic products into clinical use should be done with great care.

Kärrholm et al. [15] recommend investigation in small trials, before entering larger clinical trials, when introducing new orthopaedic products. The aim is to limit possible hazards to as few patients as possible. Thanner et al. evaluated the properties of Boneloc ${ }^{\circledR}$ and included 30 patients, of whom 14 received Boneloc ${ }^{\circledR}$. 
Table 2 Observed micromotion at 2-year follow-up, mean (SD)

\begin{tabular}{|c|c|c|c|c|c|c|}
\hline \multirow[t]{2}{*}{ Axes } & \multicolumn{3}{|c|}{ Rotation $\left(^{\circ}\right)(\mathrm{SD})$} & \multicolumn{3}{|c|}{ Translation $(\mathrm{mm})(\mathrm{SD})$} \\
\hline & $X$ & $Y$ & $Z$ & $X$ & $Y$ & $Z$ \\
\hline SmartSet HV & $-0.13(0.49)$ & $0.91(0.71)$ & $-0.08(0.18)$ & $0.08(0.11)$ & $-0.15(0.08)$ & $-0.06(0.22)$ \\
\hline Palacos R & $-0.21(0.35)$ & $1.19(0.96)$ & $-0.16(0.16)$ & $0.03(0.08)$ & $-0.16(0.06)$ & $0.02(0.23)$ \\
\hline Difference & 0.08 & -0.28 & 0.08 & 0.05 & 0.01 & -0.08 \\
\hline \multicolumn{7}{|c|}{$95 \% \mathrm{CI}$ of the difference } \\
\hline Upper & 0.42 & 0.37 & 0.21 & 0.13 & 0.07 & 0.10 \\
\hline Lower & -0.25 & -0.94 & -0.05 & -0.02 & -0.05 & -0.25 \\
\hline$P$ value & 0.60 & 0.38 & 0.20 & 0.16 & 0.74 & 0.36 \\
\hline
\end{tabular}

$\mathrm{CI}$, confidence interval

Table 3 Precision of RSA

\begin{tabular}{|c|c|c|c|c|c|c|}
\hline \multirow[t]{2}{*}{ Axes } & \multicolumn{3}{|c|}{ Rotation } & \multicolumn{3}{|c|}{ Translation } \\
\hline & $X$ & $Y$ & $Z$ & $X$ & $Y$ & $Z$ \\
\hline Mean difference of first and second reading & 0.05 & 0.07 & 0.01 & 0.00 & 0.01 & -0.01 \\
\hline $2.074 \times \mathrm{SD}$ & 0.43 & 1.01 & 0.12 & 0.10 & 0.10 & 0.22 \\
\hline
\end{tabular}

Precision values are based on 22 double examinations at 2-year follow-up

The SmartSet GHV cement was included in an in vitro study of five different PMMA bone cements [16]. Differences in viscosity behaviour, and waiting and hardening time were found between the cements investigated, leading to the conclusion of the necessity of RSA studies before any broad clinical use of new cements.

The RSA method is highly accurate and precise and therefore well suited when small study populations are involved [17]. However, small clinical trials are susceptible to biased patient selection and loss to follow-up, both experienced in the present study.

We found an almost unacceptable loss to follow-up, and retrospectively more patients should preferably have been enrolled.

Eighteen hips were initially included in each group; however, data only from 14 hips in each group were available at 2-year follow-up. Consequently there was loss of statistical power. With use of the results presented in Table 2 and Sample Power 2.0, these losses could be quantified. Differences in subsidence greater than or equal to $0.09 \mathrm{~mm}$ and/or rotation greater than or equal to $1.06^{\circ}$ could be described as statistically significant (translation along and rotations around the $Y$ axis, $\alpha=0.05$ and $\beta=0.80$, independent $t$-test). The loss of statistical power seemed to be acceptable.

The patient group at 2-year follow-up was imbalanced in gender distribution. However, we believe the gender distribution not to impair the results presented.

A large clinical study $(n=3,461)$ assessing differences between gender in clinical outcome found no such inequalities [18]. Olofsson, Önsten and Kärrholm reported no influence of patient factors such as gender on stem fixation $[9,19,20]$. A logistic regression analysis based on patient factors, type of operation and RSA data revealed that the amount of subsidence after 2 years is the best predictor of revision [2].

The distal migration of the Lubinus SP I femoral stem at 2 years predicted stem survival. Subsidence greater than $1.2 \mathrm{~mm}$ was associated with more than $50 \%$ stem revision [2].

Correlations between short-term prosthetic micromotion and future risk of prosthetic failure have been demonstrated in studies involving both hip and knee prostheses. Kärrholm et al. found it difficult to define acceptable limits of short-term prosthetic micromotion [7], while Ryd et al. [21] set the limit of distal migration at $0.2 \mathrm{~mm}$.

Micromotions of the Charnley flange 40 stem combined with Palamed $\mathrm{G}$ and Palacos $\mathrm{R}$ with gentamicin are described by Hallan et al. [13]. At 2-year follow-up, internal rotation of $1.7^{\circ}$ and $2.0^{\circ}$ was found. The subsidence was 0.18 and $0.21 \mathrm{~mm}$, respectively.

Grant et al. [22] found internal rotation of $1.1^{\circ}$ at 2-year follow-up using Charnley Elite Plus in combination with Palacos R with gentamicin. Subsidence was less than $0.18 \mathrm{~mm}$.

The levels of micromotion found in the present 2-year RSA study were similar to those presented by Grant and Hallan. There were no significant differences in either translation or rotation between SmartSet HV and Palacos $\mathrm{R}$ bone cements, the latter having documented 
good long-term clinical results [17, 23, 24]. For both cements, mean stem subsidence was smaller than $0.2 \mathrm{~mm}$, which is considered to be satisfactory with respect to longterm performance [7, 9, 22].

One hip in the Palacos $\mathrm{R}$ group rotated much more than the others: $3.67^{\circ}$ at 2 years. The subsidence for this hip at 2 years was $0.19 \mathrm{~mm}$.

Two hips in the SmartSet HV group had subsidence of $0.32 \mathrm{~mm}$ at 2 years. Data from these three hips are included in the study. Other studies have excluded such cases [13].

The internal rotation was, as expected, higher the first year than the second. Though there was no statistically significant difference in internal rotation, it seemed to be slightly smaller in the SmartSet HV group.

Our baseline RSA examinations were obtained, as recommended in Guidelines for standardisation of radiostereometry, before the patients had started the more active part of mobilisation [17]. As a consequence, we believe the results presented not to be underestimated. Those guidelines also advise double examinations to verify the precision of RSA data and that all research groups employing RSA should obtain and present their precision data. A precision of 1.0 degrees for internal rotation and $0.1 \mathrm{~mm}$ for subsidence was found in the present study. The calculation method is described in detail, since several methods have been employed for calculating such data [13, 14, 22, 25]. This makes comparison of our precision data with other publications somewhat difficult. However, we believe the method presented herein to be best suited and our precision data to be good.

The present study was dimensioned to measure movements between the implant with respect to the bone only. No attempt was made to separate possible movements between the implant and the cement mantle from movements between the cement mantle and the bone. For this reason no tantalum beads were embedded in the cement nor implanted into the cement restrictor.

Sundberg et al. and Hallan et al. deployed tantalum markers both in the bone and in the cement [13, 25]. Both studies concluded that micromovement occurred between the femoral component and the cement. The cement mantle was stable with respect to the bone. Stefánsdóttir et al. [26] described some small movement of the cement mantle, whereas movements mainly occurred inside the mantle.

Technical obstacles in employing the RSA technique to describe cement mantle movement were experienced in these studies. The tantalum markers embedded into the cement could be both poorly spatially distributed and difficult to visualise. As a consequence, a high number of patients had to be excluded when describing movement of the cement mantle.
Long-term follow-up data from clinical trials and national registry reports are needed to ensure safe practice in joint replacement. RSA studies can help to predict the long-term results when levels of micromotion are concerned.

In conclusion, the Charnley flange 40 stem in combination with the two bone cements investigated performed well at 2-year follow-up.

Acknowledgments The study was supported by a research grant from DePuy CMW Ltd., Blackpool, UK, but the authors received no direct benefits. The sponsor participated in the design of the study, but did not take part in collection, analysis or interpretation of data. The authors retained independent editorial control.

Conflict of interest None.

\section{References}

1. Buckwalter AE, Callaghan JJ, Liu SS, Pedersen DR, Goetz DD, Sullivan PM, Leinen JA, Johnston RC (2006) Results of Charnley total hip arthroplasty with use of improved femoral cementing techniques. A concise follow-up, at a minimum of twenty-five years, of a previous report. J Bone Joint Surg Am 88:1481-1485. doi:88/7/1481[pii]

2. Karrholm J, Borssen B, Lowenhielm G, Snorrason F (1994) Does early micromotion of femoral stem prostheses matter? 4-7-year stereoradiographic follow-up of 84 cemented prostheses. J Bone Joint Surg Br 76:912-917

3. Mjoberg B, Brismar J, Hansson LI, Pettersson H, Selvik G, Onnerfalt R (1985) Definition of endoprosthetic loosening. Comparison of arthrography, scintigraphy and roentgen stereophotogrammetry in prosthetic hips. Acta Orthop Scand 56:469-473

4. Thanner J, Freij-Larsson C, Karrholm J, Malchau H, Wesslen B (1995) Evaluation of Boneloc. Chemical and mechanical properties, and a randomized clinical study of 30 total hip arthroplasties. Acta Orthop Scand 66:207-214

5. Furnes O, Lie SA, Havelin LI, Vollset SE, Engesaeter LB (1997) Exeter and charnley arthroplasties with Boneloc or high viscosity cement. Comparison of 1,127 arthroplasties followed for 5 years in the Norwegian Arthroplasty Register. Acta Orthop Scand 68:515-520

6. Selvik G, Alberius P, Aronson AS (1983) A roentgen stereophotogrammetric system. Construction, calibration and technical accuracy. Acta Radiol Diagn (Stockh) 24:343-352

7. Karrholm J, Herberts P, Hultmark P, Malchau H, Nivbrant B, Thanner J (1997) Radiostereometry of hip prostheses. Review of methodology and clinical results. Clin Orthop Relat Res $\operatorname{Nov}(344): 94-110$

8. Malchau H (2000) Introducing new technology: a stepwise algorithm. Spine 25:285

9. Onsten I, Akesson K, Besjakov J, Obrant KJ (1995) Migration of the Charnley stem in rheumatoid arthritis and osteoarthritis. A roentgen stereophotogrammetric study. J Bone Joint Surg $\mathrm{Br}$ 77:18-22

10. Liu CZ, Green SM, Watkins ND, Baker D, McCaskie AW (2005) Dynamic creep and mechanical characteristics of SmartSet GHV bone cement. J Mater Sci Mater Med 16:153-160. doi:10.1007/ s10856-005-5893-y

11. d'Aubigne RM, Postel M (2009) The classic: functional results of hip arthroplasty with acrylic prosthesis 1954. Clin Orthop Relat Res 467:7-27. doi:10.1007/s11999-008-0572-1 
12. Hardinge K (1982) The direct lateral approach to the hip. J Bone Joint Surg Br 64:17-19

13. Hallan G, Aamodt A, Furnes O, Skredderstuen A, Haugan K, Havelin LI (2006) Palamed G compared with Palacos R with gentamicin in Charnley total hip replacement. A randomised, radiostereometric study of 60 HIPS. J Bone Joint Surg $\mathrm{Br}$ 88:1143-1148. doi:88-B/9/1143[pii]

14. Ranstam J, Ryd L, Onsten I (2000) Accurate accuracy assessment: review of basic principles. Acta Orthop Scand 71:106-108

15. Karrholm J, Gill RH, Valstar ER (2006) The history and future of radiostereometric analysis. Clin Orthop Relat Res 448:10-21. doi:00003086-200607000-00003[pii]

16. Kock HJ, Huber FX, Hillmeier J, Jager R, Volkmann R, Handschin AE, Letsch R, Meeder PJ (2008) In vitro studies on various PMMA bone cements: a first comparison of new materials for arthroplasty. Z Orthop Unfall 146:108-113. doi: 10.1055/s-2007-989334

17. Valstar ER, Gill R, Ryd L, Flivik G, Borlin N, Karrholm J (2005) Guidelines for standardization of radiostereometry (RSA) of implants. Acta Orthop 76:563-572. doi:V42136W7L1733G68 [pii]

18. Kostamo T, Bourne RB, Whittaker JP, McCalden RW, MacDonald SJ (2009) No difference in gender-specific hip replacement outcomes. Clin Orthop Relat Res 467:135-140. doi:10.1007/s11999-008-0466-2

19. Olofsson K, Digas G, Karrholm J (2006) Influence of design variations on early migration of a cemented stem in THA. Clin Orthop Relat Res 448:67-72. doi:00003086-20060700000011[pii]

20. Karrholm J, Anderberg C, Snorrason F, Thanner J, Langeland N, Malchau H, Herberts P (2002) Evaluation of a femoral stem with reduced stiffness. A randomized study with use of radiostereometry and bone densitometry. J Bone Joint Surg Am 84-A:1651-1658

21. Ryd L, Albrektsson BE, Carlsson L, Dansgard F, Herberts P, Lindstrand A, Regner L, Toksvig-Larsen S (1995) Roentgen stereophotogrammetric analysis as a predictor of mechanical loosening of knee prostheses. J Bone Joint Surg Br 77:377-383

22. Grant P, Aamodt A, Falch JA, Nordsletten L (2005) Differences in stability and bone remodeling between a customized uncemented hydroxyapatite coated and a standard cemented femoral stem. A randomized study with use of radiostereometry and bone densitometry. J Orthop Res 23:1280-1285. doi:S0736-0266(05) 00103-8[pii]

23. Havelin LI, Espehaug B, Vollset SE, Engesaeter LB (1995) The effect of the type of cement on early revision of Charnley total hip prostheses. A review of eight thousand five hundred and seventy-nine primary arthroplasties from the Norwegian Arthroplasty Register. J Bone Joint Surg Am 77:1543-1550

24. Herberts P, Malchau H (1997) How outcome studies have changed total hip arthroplasty practices in Sweden. Clin Orthop Relat Res Nov(344):44-60

25. Sundberg M, Besjakov J, von Schewelow T, Carlsson A (2005) Movement patterns of the C-stem femoral component: an RSA study of 33 primary total hip arthroplasties followed for two years. J Bone Joint Surg Br 87:1352-1356. doi:87-B/10/1352[pii]

26. Stefansdottir A, Franzen H, Johnsson R, Ornstein E, Sundberg M (2004) Movement pattern of the Exeter femoral stem; a radiostereometric analysis of 22 primary hip arthroplasties followed for 5 years. Acta Orthop Scand 75:408-414. doi:K0FGQT 51K6271K82[pii] 